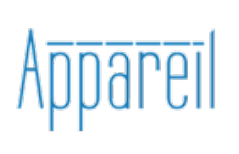

Appareil

12 | 2013

Walter Benjamin. Politiques de l'image

\title{
Benjamin : image, trace et politique
}

\section{Adolfo Vera}

\section{(2) OpenEdition}

\section{Journals}

Édition électronique

URL : http://journals.openedition.org/appareil/1946

DOI : 10.4000/appareil. 1946

ISSN : 2101-0714

Éditeur

MSH Paris Nord

Référence électronique

Adolfo Vera, « Benjamin : image, trace et politique », Appareil [En ligne], 12 | 2013, mis en ligne le 13 décembre 2013, consulté le 30 juillet 2020. URL : http://journals.openedition.org/appareil/1946 ; DOI : https://doi.org/10.4000/appareil.1946

Ce document a été généré automatiquement le 30 juillet 2020.

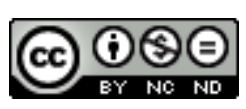

Appareil est mis à disposition selon les termes de la Licence Creative Commons Attribution - Pas d'Utilisation Commerciale - Pas de Modification 4.0 International. 


\title{
Benjamin : image, trace et politique
}

\author{
Adolfo Vera
}

1 Il faut donc reprendre la question de la trace photographique d'un point de vue politique. On peut émettre l'hypothèse que la portée essentiellement politique, pour notre époque, de la trace photographique est de permettre un type d'inscription foncièrement spectrale. Le défaut de la théorie de la photographie de Barthes, est, pour André Rouillé, de penser ce rapport spectral à partir d'une ontologie de l'image qui considère l'inscription de la trace comme fixation pérenne de l'événement ${ }^{1}$. Le " ça-aété " barthésien, en fin de compte, ne permet pas de penser le moment d'indétermination, d'indécidabilité, de dissémination propre à la trace photographique, ou, s'il le permet, c'est toujours grâce à une subordination de celui-ci par rapport à l'index, à la puissance ontologique supérieure du référent. Or, ce type d'inscription spectrale de la trace photographique a été depuis le début souligné par les commentateurs et par les photographes eux-mêmes, et le développement de la photographie spirite - avec de fameux pratiquants comme Balzac et Victor Hugo - en témoigne'. Il s'agit, d'une part, des croyances archaïques envers l'image dont parle Benjamin (la valeur cultuelle), et d'autre part, du développement des expérimentations optiques (l'anamorphose, le microscope, la fantasmagorie, etc.) qui permettront l'élaboration d'un appareil qui peut inscrire ce que la vue ne voit pas (l'inconscient optique) $)^{3}$.

2 Pour Benjamin, la trace a un rapport assez particulier avec l'aura. On peut lire dans le chapitre dédié au « Flâneur » du Livre des passages:

Trace et aura. La trace est l'apparition d'une proximité, quelque lointain que puisse être ce qui l'a laissé. L'aura est l'apparition d'un lointain, quelque proche que puisse être ce qui l'évoque. Avec la trace, nous nous emparons de la chose; avec l'aura, c'est elle qui se rend maîtresse de nous ${ }^{4}$.

3 La photographie serait, dans ce sens, un objet technique qui met en jeu d'une manière particulièrement complexe les rapports entre trace et aura.

4 Revenons à la toute fin de la Petite histoire de la photographie (1931). Dans un paragraphe aux allures prophétiques - il décrit, par exemple, le processus de miniaturisation 
progressive de l'appareil photographique, ce qui permettra de «saisir des images fugaces et cachées »- Benjamin signale :

Ce n'est pas en vain que l'on a comparé les clichés d'Atget au lieu du crime. Mais chaque recoin de nos villes n'est-il pas le lieu d'un crime? Chacun de nos passants n'est-il pas un criminel? Le photographe - successeur de l'augure et de l'haruspice - n'a-t-il pas le devoir de découvrir la faute et de dénoncer le coupable sur ses images ?

5 Ce qui est ici problématisé, c'est le rapport qu'un appareil comme la photographie peut avoir avec un phénomène que Benjamin identifie, dans plusieurs de ses écrits, comme l'un des plus marquants de la modernité capitaliste : l'effacement des traces auquel se voit voué l'homme moderne qui est, pour le dire avec Poe, un " homme de foules ${ }^{6}$ ». Dans le chapitre dédié au flâneur, dans l'essai sur Le Paris du Second Empire chez Baudelaire (1938), Benjamin écrit :

Un voile pour le flâneur recouvre cette image [de la ville]. La masse est ce voile [...]. C'est seulement lorsque ce voile se déchire et offre au regard du flâneur « une place populeuse » dont "l'émeute a fait une solitude » que lui aussi voit le visage authentique de la grande ville?

Il s'agit donc d'un rapport avec la politique comme ce qui déchire le voile de la marchandise - la fantasmagorie - qui aveugle le flâneur ; suite à ce déchirement, la ville apparaît comme un espace vide où les traces ont été effacées.

Ilaria Brocchini a souligné, dans son livre Trace et disparition ${ }^{8}$, le caractère éminemment ambigu et fort complexe de la notion de trace chez Benjamin. Il s'agit de deux mouvements où les questions (essentielles pour la pensée benjaminienne) de l'intérieur, de l'historicisme architectural (les façades historicisantes), de la fantasmagorie, entre autres, sont traitées : l'activité qui consiste à laisser des traces et celle qui consiste à les effacer. Comme l'avait déjà remarqué Georg Simmel, ce sont deux activités qu'il faut considérer à l'intérieur du processus à partir duquel l'expérience traditionnelle, celle fondée sur la transmission des récits identitaires entre les générations, c'est-à-dire l'Erfahrung, se transforme en Erlebnis, c'est-à-dire en une expérience de l'instant, du choc et de la discontinuité du fait de la vie urbaine pleine de stimuli que la conscience n'arrive pas à appréhender. Dans ce sens là, la trace acquiert un caractère éminemment conservateur, puisqu'elle essaie de réduire la violence avec laquelle, dans la vie moderne, toute tradition (l'aura, la valeur cultuelle) est effacée. Si l'habitant des métropoles modernes cherche à «laisser des traces»-jusqu'à se transformer en un "homme-étui» qui habite des maisons qui sont comme des coquilles - c'est bien parce qu'il ne peut pas supporter d'être réduit au simple Erlebnis et que des restes d'Erfahrung demeurent en lui. Brocchini propose de parler des « traces de synthèse ", "en adoptant un terme de Benjamin qui nomme "synthèse" la reconstruction, à l'époque de l'Erlebnis, des produits de l'Erfahrung ${ }^{9}$ ». Or, les totalitarismes travaillent très précisément sur ce besoin, et s'approprient des fantasmagories modernes telles que les Traumhauser ou le cinéma pour manipuler les masses et les aveugler face au fait politique de l'effacement des traces. Les totalitarismes mythologisent les traces et les font fonctionner comme des images de rêve qui renvoient à une origine intemporelle (le Volk des nazis par exemple). C'est justement ici que pour Benjamin le rôle de certains artistes est essentiel, puisqu'ils se transforment en effaceurs de traces pour montrer le mensonge de cette mythologisation. C'est le cas d'Adolf Loos, Le Corbusier ou Brecht, et à cela se réfère l'idée d'une politisation de l'art contre l'esthétisation de la politique fasciste dont parle 
Benjamin à la fin de l'essai sur la reproductibilité technique. Dans ce sens, nous voudrions émettre l'hypothèse que si la trace produite par l'appareil photographique un type d'inscription dont le caractère de fixation n'est pas plus important que celui qui concerne la manipulation ou l'effacement qu'elle peut toujours subir - a bien une portée politique, celle-ci doit être recherchée justement du côté de sa capacité à montrer le vide ou l'absence, comme le faisaient les photos d'Atget dont parlait Benjamin. Mais nous y reviendrons.

8 Avant cela, il nous faut faire encore un détour par les implications théoriques des phrases citées auparavant sur Atget. Elles nous parlent d'un certain «devoir» du photographe de découvrir le coupable du crime, qui pouvait être n'importe quel passant. En fait, il s'agit de l'importance qu'a pour Benjamin la figure du détective (imposée par le roman policier) dans le phénomène de l'effacement des traces. Dans la situation du choc subie par le passant dans les rues préhaussmanniennes, la littérature essaie, au début du xix $x^{e}$ siècle, de créer des figures rassurantes, qui en quelque sorte évitent l'imposition trop violente de l'Erlebnis. Par exemple, les physiologies qui peuplent les feuilletons et les journaux cherchent à définir des types humains propres à la ville moderne, mais qui apparaissent comme proches et non problématiques, au-delà de tout conflit social qui, on le sait bien, au contact du mélange presque inévitable de toutes les classes sociales dans la foule, risque à chaque instant d'éclater. L'apparition du roman policier, selon Benjamin, obéit à l'échec que ces formes littéraires subissent du fait de leur incapacité à représenter la complexité grandissante de la vie des habitants des villes modernes. Il n'est pas un hasard du tout que ce soit Baudelaire qui ait traduit le grand inventeur du genre, Edgar Poe. Tous les deux ont très vite compris que la masse permettait l'apparition des conditions idéales pour commettre un crime. «La masse [écrit Benjamin dans Le Paris du Second Empire chez Baudelaire] apparait ici comme l'asile qui protège l'asocial de ses poursuivants $[. . .]^{10}$ ». Et il continue : «En ces temps de terreur où chacun tient par quelque chose du conspirateur, chacun peut également se trouver conduit à jouer au détective. La flânerie lui offrira les meilleures perspectives ${ }^{11}$ ». Il n'y a que des traces face au flâneur-détective, des traces qui apparaissent et disparaissent, qui s'évanouissent juste quand on commence à les suivre. Chaque trace signale un crime, toujours sous la lumière à gaz et la splendeur de la fantasmagorie parisienne. Dans ces conditions, il n'est pas si difficile de disparaitre. Ce n'est pas dans un autre sens qu'il faut comprendre l'affirmation de Benjamin : «Le contenu social primitif du roman policier est l'effacement des traces de l'individu dans la foule de la grande ville ${ }^{12} »$. Or, à ce mouvement d'effacement violent des traces dans la rue, s'oppose la volonté inverse de conserver, à l'intérieur, la plus grande quantité possible de traces. Ce phénomène est décrit par Benjamin dans le chapitre «L'Intérieur, la trace » du Livre des passages ${ }^{13}$. Un passage du texte sur Baudelaire déjà cité résume l'enjeu:

On perçoit depuis Louis-Philippe dans la bourgeoisie un effort pour se dédommager du peu de traces que laisse la vie privée dans la grande ville. Elle cherche ce dédommagement entre ses quatre murs. Tout se passe comme si elle mettait un point d'honneur à sauver de la disparition dans l'éternité des siècles, sinon son existence terrestre, du moins ses articles d'usage courant et ses accessoires. Elle prend l'empreinte d'une foule d'objets; elle cherche des fourreaux et des étuis pour les pantoufles et pour les montres, pour les thermomètres et les coquetiers, pour les couverts et les parapluies. Elle préfère les housses de peluche et des velours qui conservent l'empreinte de chaque contact. Avec le style Makart - le style du Second Empire finissant -, l'appartement devient une sorte de coquille. Ce style considère 
que l'appartement représente l'enveloppe de l'individu et il l'y dépose avec tous ses accessoires, gardant ainsi fidèlement la trace comme la nature conserve dans le granit celle d'une faune disparue ${ }^{14}$.

9 Cependant, à l'extérieur même, dans l'espace public où la loi doit légiférer, une suite de mesures de contrôle policier commence à apparaître pour contrarier ou, pourrait-on dire, au moins équilibrer ce processus d'effacement des traces. Benjamin signale comment à partir de 1805 l'administration de Napoléon impose la numérotation des maisons et des immeubles parisiens. Mais d'autres mesures touchent directement la photographie, notamment celles qui concernent la méthode d'identification de Bertillon (le procédé connu sous le nom d'«anthropométrie judiciaire »). Il s'agissait d'établir, dans une continuité plus ou moins évidente avec les recherches de la physiognomonique et de la phrénologie, une cartographie très précise du visage humain. Contre le caractère démoniaque et l'inquiétante étrangeté qui, surtout sous la faible illumination du gaz, attendent toujours le passant des rues des villes modernes, la police essaie de raffiner technologiquement les modalités de l'identification rationnelle. Bertillon contre Atget: si celui-là cherche à faire apparaître jusqu'au dernier secret d'une supposée identité culturelle ou même raciale dans la surface du visage humain, pour clarifier le mal et saisir - en vertu de la lumière inscrite comme trace photographique - l'obscurité démoniaque du crime (à la manière des physiologistes du début du siècle), celui-ci a montré le vide de la ville, comme ce qui apparaît une fois le voile (la masse) déchiré. Dans ce sens, Benjamin écrit :

La photographie permet pour la première fois de fixer durablement et sans aucune ambiguïté les traces d'un homme. Le roman policier naît au moment où était assurée cette conquête, la plus décisive de toutes, sur l'incognito de l'homme. Il n'est pas possible depuis lors d'envisager quelque fin à ses efforts pour s'emparer de celui-ci et le figer dans ses paroles et ses actes ${ }^{15}$.

10 Cependant, il n'y a pas lieu d'assimiler la position du photographe avec celle du détective ni avec celle du criminel. Il agit dans une zone intermédiaire qui, en revanche, est bien celle du flâneur. Cette zone a été traitée longuement par Siegfried Kracauer dans son essai de 1925 sur le Roman policier ${ }^{16}$. Ce "traité philosophique", comme on le sait, considère, à la différence de l'approche plutôt sociologique de Benjamin, ce genre littéraire d'un point de vue métaphysique. Ce que manifeste le roman policier selon Kracauer est la crise radicale de la ratio et le vide existentiel auquel se voit condamné l'homme des villes modernes, jeté dans ce qu'il définit comme le «monde inférieur ", le monde de la matérialité (de la marchandise), sans pouvoir réaccéder au niveau supérieur, celui du sacré. Le «mystère » qui se présente sous la modalité de l'embrouillement des traces dans le roman policier est la manifestation de l'obscurité de ce qui échappe à la raison (et qui prend la figure du mal et du crime). Le détective n'est donc que le dernier représentant d'une ratio dont les réalisations apparaissent mécaniquement, sans contenu ni sens (sous la modalité de l'Erlebnis et non pas de l'Erfahrung). Obsédé par le calcul, pour ne pas laisser échapper les pouvoirs de la raison mathématique, il prend la place du prêtre à l'époque de la mort de dieu. Il dit la messe dans les halls d'hôtel, et son dieu est la déduction. Le hall d'hôtel - si souvent représenté dans les romans policiers - a pour Kracauer une valeur symbolique essentielle. C'est l'espace qui apparaît dans son vide dénué de sens, une fois que les êtres humains n'ont plus la capacité de constituer des lieux, puisqu'il n'y a plus de communauté humaine. "Lorsque les hommes [écrit-il] ont dénué la relation constitutive du lieu, celui-ci ne conserve qu'une valeur décorative ${ }^{17}$ ». Et si pour Kracauer le hall d'hôtel est le pendant de l'église, il faut quand même considérer que 
dans celui-ci ne se rassemble plus une communauté, mais plutôt le «public », dont la loi est l'anonymat.

Mais alors que l'église est destinée au service de Celui chez qui on se rend, le hall d'hôtel est au service de tous ceux qui s'y rendent pour n'y rencontrer personne. C'est la scène de ceux qui ne cherchent ni ne trouvent Celui que l'on recherche toujours, et qui par conséquent sont des hôtes de l'espace comme tel, de l'espace qui les entoure et qui n'a d'autre destination que de les entourer ${ }^{18}$.

11 En lisant ces lignes, on ne peut pas s'empêcher de penser à la peinture d'Edward Hopper, mais aussi à la série The Americans de Robert Frank.

Pour Kracauer, ce serait une erreur de classer le roman policier à partir des catégories romantiques. Si le mal, le démoniaque et le crime - sous le signe du «mystère »-y apparaissent, c'est néanmoins sous le contrôle de la légalité, dont l'abstraction fait du crime une autre manifestation de la loi. Le crime est une excuse pour faire valoir les droits de la police. L'inquiétante étrangeté qui se manifeste dans le roman policier n'est plus celle des romantiques (Hoffmann notamment), qui appartiendrait selon lui à la sphère supérieure (le religieux) - elle agit dans la sphère inférieure, celle de la marchandise. Mais celle-ci conserve encore un trait de celle-là: son caractère d'interruption. Selon Kracauer :

Un long chemin sépare le méchant glorifié dans les romans anciens, de l'adversaire redouté de Sherlock Holmes. Dans le premier cas, l'acte est dérivé d'une passion personnelle, si faussement sentimentale que soit sa représentation; dans le cas présent, le démonisme pâlit devant l'éclat des déductions; en démêlant les faits, elles tirent et distordent à la lumière grise du jour l'origine de l'effet démoniaque. Dans le premier cas, l'être coupable possède une force magique indestructible ; dans le second, la magie est une caractéristique illusoire qui ne peut tromper qu'aussi longtemps que l'inexplicable n'est pas maîtrisé par la logique. L'inquiétante étrangeté n'est, elle non plus, ni un attribut de l'esprit ténébreux qui renvoie audelà de la familiarité de l'espace habité, ni attachée à l'acte qu'elle ne précipite pas dans le royaume des ténèbres - elle provient du caractère énigmatique d'un fait qui, en tant que point ultime d'enchaînements inexplorés, arrête brusquement le flux ininterrompu de l'agitation sociale ${ }^{19}$.

13 L'interruption de ce flux social, celui des masses, est ce qui est mis en évidence par le roman policier. L'imaginaire du détective, du criminel, mais aussi le hall d'hôtel et les espaces vides peuvent être définis comme autant de manifestations de l'effacement progressif des traces des lieux pour permettre l'apparition des espaces vidés de toutes traces. On peut ajouter : un lieu est un espace où l'on peut identifier des traces. Celles-ci disparues, il ne reste que de l'espace. C'est le processus de cette disparition qui est mis en œuvre dans le roman policier.

Mais revenons à la photographie. En considérant cette dernière remarque de Kracauer, et en reprenant les analyses faites par Max Milner, dans son essai sur l'optique fantastique ${ }^{20}$ où la surabondance de miroirs, de lorgnettes et d'effets littéraires adoptés comme autant d'effets d'optique est rattachée à une subjectivité qui se veut disloquée, mais qui n'en reste pas moins subjective, on pourrait faire l'hypothèse que la photographie n'est pas un appareil à l'usage romantique justement parce qu'elle apparaît à une époque où la subjectivité est transformée en marchandise. C'est justement en ceci que la photographie n'appartient plus au régime autonome de l'art. 
«La tentative [écrit Benjamin dans le Livre des passages] pour provoquer une confrontation systématique de l'art et de la photographie était d'abord vouée à l'échec. Il fallait qu'elle fût un moment de [la] confrontation de l'art et de la technique, accomplie par l'histoire ${ }^{21} »$. Or, à l'époque où les foules agissent mécaniquement, comme le décrit Poe dans L'homme des foules, où, comme le signale Baudelaire, la vertu sociale la plus haute n'est autre que la prostitution, le caractère de «reproduction mécanisée » (présent déjà dans un dispositif comme le physionotrace) devient essentiel et nous oblige à nous méfier des catégories trop romantiques comme celle de l'inquiétante étrangeté. Ou, comme le faisait Kracauer, nous oblige à les limiter aux phénomènes d'interruption des flux sociaux.

La portée politique de la trace photographique doit donc être considérée par rapport au caractère éminemment technique de l'image photographique. Si toute image est un type particulier d'inscription, et donc de trace, celle qui correspond à l'appareil photographique est avant tout reproductible. De ce fait, elle correspond comme aucune autre à une époque, celle décrite par Baudelaire, où, comme le disait Benjamin, même les singularités les plus excentriques ne peuvent pas rompre avec le «cercle magique du type ». L'automatisme des foules correspond directement à la mécanisation de l'image photographique. Cette mécanisation concerne l'apparaître et le disparaître de la trace dans un monde dominé par la fantasmagorie de la marchandise. Il s'agit donc de l'expérience du choc, qui, selon Benjamin, implique la progressive importance de la mémoire volontaire (une "conscience perpétuellement aux aguets", qui n'est plus capable d'accueillir toutes les traces renvoyées par les objets), par rapport à la mémoire involontaire, celle qui permet l'expérience authentique ${ }^{22}$. Selon Benjamin :

La discontinuité des moments du choc trouve sa cause dans la discontinuité d'un travail devenu automatique, n'admettant plus l'expérience traditionnelle qui présidait au travail artisanal. Au choc éprouvé par celui qui flâne dans la foule correspond une expérience inédite : celle de l'ouvrier devant la machine ${ }^{23}$.

La photographie aura donc été au cœur des causes historiques qui expliquent la "déchéance de l'aura », c'est-à-dire, selon Benjamin, des "souvenirs plus ou moins distincts dont est imprégnée chaque image qui surgit du fond de la mémoire involontaire ${ }^{24} »$. La trace photographique, qui surgit comme le type d'inscription propre à une époque qui se caractérise par le phénomène généralisé de l'effacement des traces (et ici la stratégie de la disparition politique, utilisée par tous les régimes totalitaires, doit apparaitre comme l'horizon théorique d'une philosophie politique de la trace), est à la base de la déchéance d'une capacité psychique, celle qui permettait d'investir les choses du pouvoir de lever le regard. Ce que l'on a défini comme le caractère «spectral » de la trace y est supposé : il s'agit d'un processus par lequel les objets peuvent s'inscrire dans la mémoire justement parce qu'ils n'existent pas en euxmêmes dans un Présent de la perception, mais conformément à la logique (définie par Freud) de l'après-coup. Or, cette inscription, à l'époque du choc, n'est possible que grâce aux appareils comme le cinéma ou la photographie, qui sont, selon Benjamin, des «moyens d'immunisation ${ }^{25}$ ».

L'anachronisme - la non-coïncidence du temps avec soi-même- de ce type d'inscription, anachronisme qui définit aussi la condition spectrale, a été décrit par Benjamin dans un passage d'Enfance berlinoise, où il traite du déjà vu. Le déjà vu ne définit pas uniquement le passé qui revient, mais aussi l'avenir qui a laissé ses traces dans le passé. 
On a souvent décrit le déjà vu. Est-ce que la formule est vraiment heureuse ? Ne faudrait-il pas parler d'événements qui nous parviennent comme un écho dont l'appel qui lui donna naissance semble avoir été lancé un jour dans l'obscurité de la vie écoulée? Au reste, à cela correspond le fait que le choc, par lequel un instant se donne à notre conscience comme déjà vécu, la plupart du temps nous frappe sous la forme d'un son [...] Étrange qu'on n'ait pas encore suivi le trajet inverse de cet éloignement - le choc par lequel un mot nous fait trébucher comme un manchon oublié dans notre chambre. De même que celui-ci conduit notre pensée vers une étrangère qui était là, il y a des mots ou des silences qui conduisent notre pensée vers cette étrangère invisible : l'Avenir, qui les oublia chez nous [... $]^{26}$. arrive est archivé (déjà par la pensée elle-même, dont la mémoire comme inscription de l'événement dans la temporalité de l'après-coup est le modèle). Et l'archive spectralise l'événement, puisqu'il le transforme en trace (en document) : inscription qui par le fait d'apparaître peut toujours disparaître - et c'est bien là l'enjeu de la dialectique entre trace et aura chez Benjamin, mais aussi de la portée philosophique du roman policier chez Kracauer, et de la critique de la «métaphysique de la présence » chez Derrida, puisque la trace - la différance comme production du différer - n'apparaît qu'en tant qu'elle peut disparaître. On peut faire l'hypothèse que cette époque produit des appareils dont la capacité de spectralisation de l'événement se complexifie et augmente à chaque fois (à chaque innovation technique). Si la trace photographique impliquait déjà une forte spectralisation de ce qui apparaît, du fait du flou de l'image et de la possibilité de sa manipulation (et de là la réception immédiatement "spiritiste " de cet appareil, chez Balzac, Victor Hugo et bien d'autres), la trace cinématographique est encore moins solide et plus fantomatique du fait de l'incapacité dans laquelle se trouve la conscience de capturer le mouvement excessivement rapide des images fixes. Aujourd'hui, il nous faudrait donc penser comme une sorte de consolidation de la "puissance de spectralité " propre aux appareils l'arrivée du numérique, et l'on pourrait comprendre dans ce sens les propos de Derrida dans le film Ghost Dance (1983) de Ken Mac Mullen :

Alors que je crois au contraire que l'avenir est aux fantômes et que la technologie moderne de l'image, de la cinématographie, de la télécommunication décuple le pouvoir des fantômes, du retour des fantômes. 


\section{BIBLIOGRAPHIE}

Baudelaire Charles, «Quelques caricaturistes français », in Euvres complètes, Paris, Le Dantec (La Pléiade), 1955, p. 994-1013.

Benjamin Walter, Enfance Berlinoise, Jean Lacoste (trad.), Paris, Maurice Nadeau, 1978.

Benjamin Walter, Paris, capitale du XIX $X^{e}$ siècle. Le livre des Passages, Jean Lacoste (trad.), Paris, Éditions du Cerf, 1989.

Benjamin Walter, «Le Paris du Second Empire chez Baudelaire », in Charles Baudelaire, Jean Lacoste (trad.), Paris, Payot, 1990.

Benjamin Walter, « À propos de quelques motifs baudelairiens », in Écrits français, Paris, Gallimard, 1991.

Benjamin Walter, « L'œuvre d'art à l'ère de sa reproduction mécanisée », in Écrits français, Paris, Gallimard, 1991.

Benjamin Walter, Petite histoire de la photographie, Paris, Allia, 2012.

Brocchini Ilaria, Trace et disparition, Paris, L'Harmattan, 2006.

Kracauer Siegfried, Le roman policier. Un traité philosophique, Rainer Rochlitz (trad.), Paris, Payot, 2001.

Krauss Rosalind, «Sur les traces de Nadar ", in Le photographique. Pour une théorie des écarts, Paris, Macula, 1990, p. 18-36.

Milner Max, La fantasmagorie. Essai sur l'optique fantastique, Paris, PUF, 1982.

Nadar, «Quand j'étais photographe », in Dessins et écrits, t. II, Paris, Arthur Hubschmid, 1979, p. 967-1284.

Poe Edgar Allan, « L'homme des foules », in Euvres en prose, Charles Baudelaire (trad.), Paris, Le Dantec (La Pléiade), 1951, p. 311-320.

Rouillé André, La photographie. Entre document et art contemporain, Paris, Gallimard, 2005.

Thélot Jérôme, Les inventions littéraires de la photographie, Paris, PUF, 2003.

\section{NOTES}

1. André Rouillé, La photographie. Entre document et art contemporain, Paris, Gallimard, 2005.

2. Jérôme Thélot, Les inventions littéraires de la photographie, Paris, PUF, 2003. En ce qui concerne l'attitude de Balzac et sa fameuse "Théorie des spectres", on peut également consulter le premier chapitre des mémoires de Nadar, «Quand j'étais photographe », in Dessins et écrits, t. II, Paris, Arthur Hubschmid, 1979, p. 967-1284 ; également, l'essai de Rosalind Krauss, "Sur les traces de Nadar ", in Le photographique. Pour une théorie des écarts, Paris, Macula, 1990, p. 18-36.

3. Max Milner, La fantasmagorie. Essai sur l'optique fantastique, Paris, PUF, 1982.

4. Walter Benjamin, Paris, capitale du XIXe siècle. Le livre des Passages, Jean Lacoste (trad.), Paris, Éditions du Cerf, 1989, p. 464.

5. Ibid., p. 29.

6. Edgar Allan Poe, «L'homme des foules », in CEuvres en prose, Charles Baudelaire (trad.), Paris, Le Dantec (La Pléiade), 1951, p. 311-320. 
7. Walter Benjamin, «Le Paris du Second Empire chez Baudelaire», in Charles Baudelaire, Jean Lacoste (trad.), Paris, Payot, 1990, p. 91. Les passages entre guillemets sont extraits de l'essai «Quelques caricaturistes français» de Baudelaire. Voir Euvres complètes, Paris, Le Dantec (La Pléiade), 1955, p. 994-1013.

8. Ilaria Brocchini, Trace et disparition, Paris, L'Harmattan, 2006.

9. Ibid., p. 14.

10. Walter Benjamin, « Le Paris du Second Empire chez Baudelaire », p. 64-65.

11. Ibid., p. 65.

12. Ibid., p. 69.

13. Walter Benjamin, « L'intérieur, la trace », in Paris, capitale du XIX siècle, p. 230-246.

14. Ibid., p. 72-73.

15. Ibid., p. 75.

16. Siegfried Kracauer, Le roman policier. Un traité philosophique, Rainer Rochlitz (trad.), Paris, Payot, 2001.

17. Ibid., p. 75.

18. Ibid., p. 76.

19. Ibid., p. 132.

20. Max Milner, La fantasmagorie. Essai sur l'optique fantastique.

21. Walter Benjamin, Paris, capitale du XIX $x^{e}$ siècle, p. 689.

22. Walter Benjamin, "À propos de quelques motifs baudelairiens », in Écrits français, Paris, Gallimard, 1991, p. 244 et suiv.

23. Ibid., p. 245.

24. Ibid.

25. Walter Benjamin, «L'œuvre d'art à l'ère de sa reproduction mécanisée », in Écrits français, p. 163. Voir aussi «Panorama impérial » et «Téléphone» dans Enfance Berlinoise, Jean Lacoste (trad.), Paris, Maurice Nadeau, 1978, p. 33-35, 39-40.

26. Walter Benjamin, «L'annonce d'une mort », in Enfance Berlinoise, p. 53.

27. Walter Benjamin, « La loutre », in Enfance Berlinoise, p. 60.

\section{RÉSUMÉS}

Si l'on part du constat que «l'effacement des traces" est l'un des effets les plus cruellement marquants des pratiques totalitaires, une philosophie politique attentive aux conséquences de la violence extrême devra nécessairement se pencher sur la question de la trace. Nous étudierons ici cette question par rapport à la théorie benjaminienne de la photographie, pour essayer de comprendre ce qui est énoncé à la fin de la Petite histoire de la photographie, au moment où Benjamin, en parlant des photos d'Atget, annonce la portée politique de la photographie à l'époque moderne : reconfigurer la scène d'un crime qui a été commis, et dont les traces ont été brouillées.

\section{INDEX}

Mots-clés : trace, image, violence, inscription, photographie 
AUTEUR

ADOLFO VERA

Docteur en philosophie à l'université Paris VIII Vincennes Saint-Denis. Professeur à l'Institut de philosophie, université de Valparaíso, Chili. 